\title{
Three-dimensional reconstruction of the oscillatory free-surfaces of a flow over antidunes: stereoscopic and velocimetric techniques
}

\author{
D. Douxchamps ${ }^{1}$, D. Devriendt ${ }^{2}$, H. Capart ${ }^{2,3}$, C.Craeye ${ }^{1,4}$, B. Macq ${ }^{1}$ and Y. Zech ${ }^{2}$ \\ ${ }^{1}$ UCL Telecom. Lab., Place du Levant, 2, B-1348 Louvain-la-Neuve, Belgium \\ ${ }^{2}$ UCL Civ. Eng. Dept., Place du Levant, 3, B-1348 Louvain-la-Neuve, Belgium \\ ${ }^{3}$ Fond National de la Recherche Scientifique, Belgium \\ ${ }^{4}$ TUE/Electromagn. Lab., PO 513, MB5600 Eindhoven, The Netherlands
}

\begin{abstract}
We present imaging methods developed to characterize the oscillatory free-surface of rapid flows and apply them to torrential currents propagating over sediment antidunes. The aim is to obtain high-resolution relief maps of the free-surface topography, in order to highlight the regular spatial patterns associated with the bedforms. Two measurement principles are outlined and tested, both based on the imaging of floating tracers dispersed on the rapidly flowing surface. The first relies on direct stereoscopic measurements obtained using two cameras, while the second exploits an original velocimetric principle allowing to derive elevation from the velocity field acquired using a single camera. The measurement procedures and image analysis algorithms are introduced for the two methods, along with the physical assumptions underlying the velocimetric principle. The results of the two techniques are compared for different free-surface patterns and good correspondence is obtained. The obtained relief maps vividly depict the variety of motifs that can evolve as a result of interaction between shallow flows and loose sediment beds.
\end{abstract}

\section{INTRODUCTION}

Of interest to hydraulic engineers and geomorphologists, bedforms of various types are observed in a wide range of modern flow environments and ancient sediment deposits. In the present paper, we focus on one of the most evanescent family of bedforms: antidunes. This class of fluvial bedforms [1],[2] appears when rapid, shallow currents propagate over coarse granular material, and is characterised by in-phase coupling between the oscillatory sediment bed and the flow free-surface. They can be observed both in the laboratory [4] and in the field [3]. Among other features, antidunes are notable for the fact that they vanish rapidly once the flow wanes. As a result, they leave few lasting traces aside from bedding and grain-sorting effects. Their geometry, in particular, has to be studied when the flow is active.

Long- or short-crested, arranged in regular arrays or in isolated trains of peaks and troughs, antidunes come in a variety of patterns. In the present study, a characterisation of such patterns is sought through measurements of the water free-surface topography. Visual access to the underlying sediment bed surface is impaired by the rough and shallow character of the flowing water layer, hence no direct measurement of the bottom topography is possible. Since the two surfaces are locked in phase with each other, however, the water surface topography provides an indirect image of the bedform pattern.

Measurements of water surface topography most often involve point sensors, placed in multisensor arrays at fixed locations or scanned across the surface. Sensors used to measure water elevation include resistance gauges, pressure transducers and acoustic beams. Resistance gauges are inapplicable in the present case because of the high sensitivity of antidune flows to intruding objects. More generally, the spatial and temporal resolution of point sensors is limited by the number of available devices or the time required to scan a sensor across the field of interest. This makes them unsuitable for the transient (on a time scale of a few seconds), spatially varied surfaces of flows over evolving bedforms. Imaging techniques, by contrast, permit non-intrusive, fast whole-field measurements (a review of velocimetric methods is given in [5]). In the present work, two distinct methods have been developed for the rapid profiling of a highly varied water surface. Both are based on the imaging of floating tracers dispersed on the flowing water, but the two rely on different reconstruction principles. The first is a stereoscopic technique, based on the matching of particles on image pairs acquired from different viewpoints using two cameras. The second is a monoscopic technique, based on particle tracking measurement of the velocity field in the horizontal plane. A Bernoulli-type principle derived from the fluid mechanics of the water free-surface is then exploited to reconstruct the vertical elevation map. As the two techniques are based on different principles and complete separation was maintained between the two data elaboration processes, intercomparison of results makes it possible to estimate the measurement quality.

The paper is organised as follows. The experimental setup used for the measurements is first presented. Each of the two methods is then detailed. Finally, results of the two methods are shown and compared.

\section{EXPERIMENTAL SETUP}

Experiments are conducted in a hydraulic flume having the following dimensions: length $=6 \mathrm{~m}$; width $=50 \mathrm{~cm}$; sidewall height $=50 \mathrm{~cm}$. The flume is tilted to obtain a bottom slope of $1 \%$. A $5 \mathrm{~cm}$ deep layer of loose sediment covers the flume bottom and is replenished during flow by means of a silo. A coarse sand of nearly uniform size distribution is used as sediment material. For the preliminary experiments presented herafter, the water inflow was not tightly controlled 


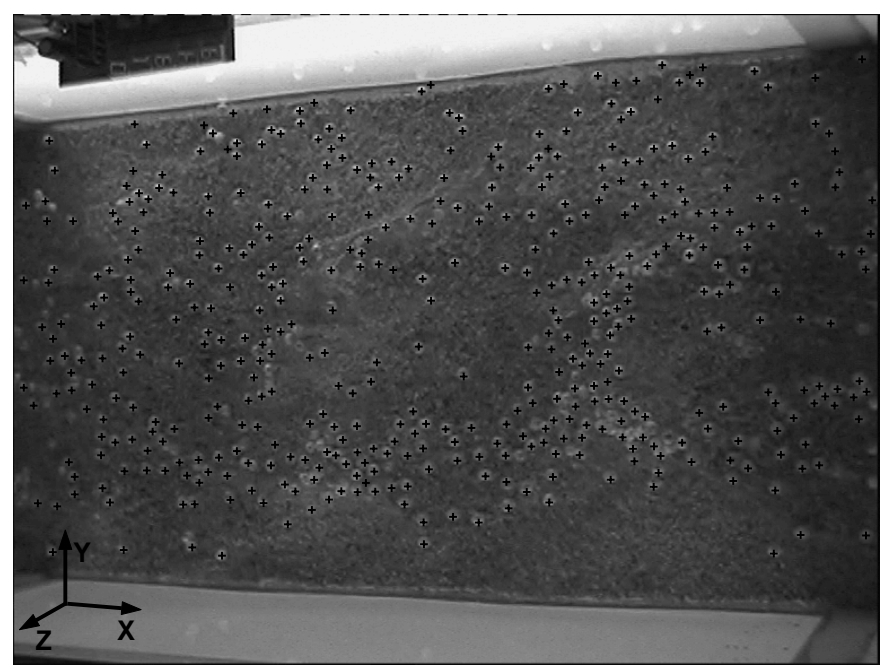

Figure 1: A snapshot from one of the stereo cameras, with the three-dimensional frame of reference. The white particles on the image are the floating tracers. Symbols (+) mark the detected particles. Flow is from left to right.

but rather freely operated in such a way as to produce a variety of patterns. Water discharges in the flume ranged from 15 to $35 \mathrm{l} / \mathrm{s}$, yielding flow depths of 4 to $6 \mathrm{~cm}$ and Froude numbers in the range $\mathrm{Fr}=1.4$ to 2 . For such high Froude numbers, the water free-surface responds strongly to the underlying oscillatory topography of the bedforms, up to the point of breaking at the wave crests when antidunes are fully developed. Starting from a plane bed, the antidunes emerge as longitudinal trains of crests and throughs initiating from downstream but stationary in phase. The antidunes are observed to respond to transient changes in the flow rate (both increase and decrease) by temporarily growing in amplitude. Amplitude responses and gradual shifts in pattern occur on a time scale of tens of seconds. By contrast, on the shorter timescale corresponding to the image acquisition (of the order of $2 \mathrm{~s}$ ), the hydrodynamics can be assumed to be quasi-steady and this is exploited hereafter to derive a single surface from each measurement sequence. Slight unsteady pulsations of the flow are however observed, and this physical source of noise will affect the results concurrently with measurement errors.

The measurement section is placed some $2 \mathrm{~m}$ upstream of the flume outlet. Moments before image acquisition, a uniform dispersion of floats is dropped onto the mean surface by means of staggered metal meshes. The tracer particles are white wooden pearls $9 \mathrm{~mm}$ in diameter. Image sequences are obtained using digital cameras placed above the flow. The velocimetric and stereoscopic methods require rather different acquisition systems, hence some care is necessary in order to operate them simultaneously to image the same scene. Two commercial digital cameras (miniDV, PAL, 25 frames per second (fps)) are used for the stereoscopic measurements. These cameras offer good image resolution ( 576 by 768 pixels) but cannot be synchronised with each other during the acquisition. To avoid motion-stereo ambiguity, it is thus necessary to synchronise them a posteriori using an interpolation procedure (see below).
The stereo cameras are placed above the flow with oblique optical angles contained in a vertical plane parallel to the direction of flow (see Fig. 1 for a sample image). For the velocimetric measurements, a high frame rate camera (250 fps) is placed directly over the flow with a nearly vertical optical axis. Due to the high frame rate, this camera requires strong lighting, obtained with four $2 \mathrm{KW}$ light sources. Such powerful lighting saturates the commercial cameras even at maximum shutter speed, and these have to be fitted with dimming filters. After positioning, the viewpoints of all three cameras are determined by placing a calibration target in their common viewing volume. This is essential for stereo reconstruction and allows the results of the two methods to be obtained in the same three-dimensional referential.

\section{STEREOSCOPIC TECHNIQUE}

In the first measurement technique, a stereoscopic procedure is applied to the dispersion of floating tracers imaged by the two commercial cameras. These recognisable feature-points are detected on the two different views and matched in order to derive their three dimensional coordinates. This requires precise synchronisation of the two views, achieved here by an interpolation procedure performed a posteriori. Finally, the water elevation map is obtained by fitting a surface through the collection of points positioned in space. Presented in more detail in [6], these various steps are outlined in the next paragraphs.

\section{A. Particles detection}

The detection process is based on a transformation of the original image with a variation of the wavelet transform [7], [8] presented in [9] and modified for our purposes. Strickland developed in this paper an optimal filter for breast tumor detection: a case where the texture of the images are surprisingly close to our images of floating particles over blurred sediments.

Since the particles have a unique known size, we can use a single set of two adapted wavelet filters instead of a complete recursive filter bank. Filtering the image along its two dimensions with the two different filters provides four filtered images: $d_{H H}, d_{L L}, d_{H L}$ and $d_{L H}$ where the indices " $H$ " and " $L$ " refer to the filter used (High- or Low-pass). While the $d_{H H}$ image could be used alone for detection, we prefer to recombine it with the $d_{L H} \cdot d_{H L}$ image to lower the effect of high-frequency noise. The combination of the different transforms is then

$$
T=\frac{1}{2}\left(\sqrt{d_{H L} \cdot d_{L H}}+d_{H H}\right),
$$

where all operators are applied element by element. The maxima of $T$ represent the particles positions and are detected using a classic neighborhood zeroing technique with an additional low-derivative constrain. The set of detected particles is shown on Fig. 1. 


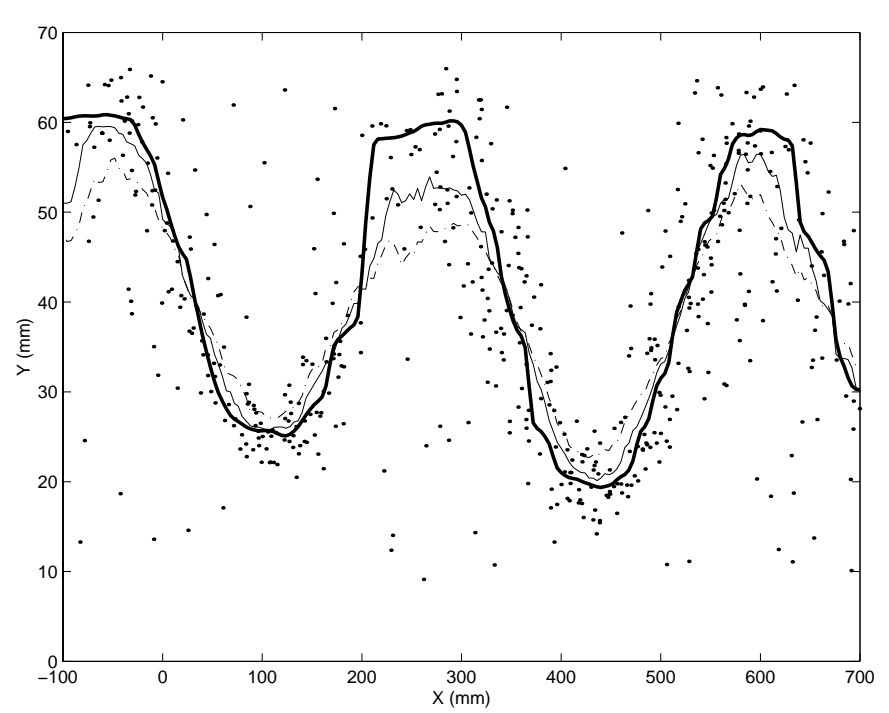

Figure 3: Sample surface slice (centerline of run 3) showing the 3D stereo points and the surface estimates obtained with different averaging methods. Dashed line: mean; continuous line: median; thick line: Bayesian estimate.

arbitrary locations. In practice, dispersion arises because of physical fluctuations, mismatches, and limited accuracy of the particle positioning and ray intersection.

Mismatching of stereo pairs constitute a particularly problematic source of error because it tends to scatter the resulting spurious points uniformly in the viewing volume rather than in a neighbourhood of the actual surface. The procedure adopted consists in binning the data into rectangular cells in the $(x, y)$ plane, then averaging the data of each bin to obtain a value of the surface elevation. To deal with mismatch error, Bayesian averaging [13] is performed for each bin. It consists in a recursive procedure which assigns to each point a probability that it constitutes a correct match, then obtains the free-surface position as an average weighted by this probability. In Fig. 3, it can be seen that this special average yields better results than both the mean and median estimates in the present case.

Another issue concerns the size of the cells to be used in the binning procedure. If the cells are too large, attenuation of the surface amplitude variations occurs, while if the cells are too small, the noise is insufficiently filtered. Both these effects can be approximately estimated from the data, and we utilise a bin size of 50 to $100 \mathrm{~mm}$ which minimises the sum of the two errors. For the stereo procedure, this results in a combined error which is estimated to be of the order of $1 \mathrm{~mm}$ (but which can be considerably larger in zones of the flow were few particles are present). This is reasonably small compared to the elevation range, which is of the order of $30 \mathrm{~mm}$.

\section{Velocimetric TECHNiQUe}

The second technique involves the reconstruction of the surface elevation map from the horizontal velocity field, by means of a relation derived from the dynamics of the fluid free-surface. A raw velocity field is first extracted from monoscopic images using a pattern-based particle tracking velocimetry (PTV) algorithm. The elevation field is then obtained after suitable along-streamline and transverse averaging of the individual velocity vectors. These steps are sketched in the next paragraphs. For more detail, the reader is referred to [14] and [12].

\section{A. Free-surface dynamics}

To describe the local dynamics of the fluid free-surface, we make the following two basic assumptions: (i) the time evolution of the loose sediment bed is sufficiently slow that it appears stationary to the rapidly flowing fluid, hence the flow can be taken as quasi- steady; (ii) the flow can be considered inviscid (but it does not have to be assumed irrotational).

Under these assumptions, and if the slope of the free-surface is everywhere moderate, then the following equations hold along a surface streamline:

$$
\begin{gathered}
\frac{\mathrm{d}}{\mathrm{d} \xi}\left(\frac{\mathbf{u} \cdot \mathbf{u}}{2}+g \eta\right)=0 \\
w=\sqrt{u^{2}+v^{2}} \frac{\mathrm{d} \eta}{\mathrm{d} \xi}
\end{gathered}
$$

where $\mathbf{x} \equiv(x, y, z)$ is the position given by its longitudinal, transverse, and vertical coordinates, $\mathbf{u} \equiv(u, v, w)$ is the fluid velocity given by its three components, $\eta(x, y)$ is the elevation of the free-surface, and $\xi$ is the curvilinear position coordinate along the horizontal plane projection of the streamline $(x(\xi), y(\xi))$ defined by

$$
\frac{\partial x}{\partial \xi}=\frac{u}{\sqrt{u^{2}+v^{2}}}, \quad \frac{\partial y}{\partial \xi}=\frac{v}{\sqrt{u^{2}+v^{2}}} .
$$

Dynamic equation (8) is the Bernoulli equation, describing the conservation of energy for a steady flow with vorticity and written for a surface streamline, while kinematic equation (9) expresses the constraint by which an infinitesimal fluid parcel belonging to the free-surface remains at the free-surface [15], [16].

From equations (8) and (9), it is at once apparent that if the horizontal velocity field $\{u, v\}(x, y)$ is known, then an ordinary differential equation can be solved for the free-surface elevation $\eta$ along each streamline. This constitutes the basis of the velocimetric technique proposed here for the measurement of free-surface topography. On a streamline-by-streamline basis, the above principle can be applied to a wide class of flows.

An especially simple situation arises if, as in the present case of antidune flow, the free-surface can be approximated as a superposition of small amplitude oscillatory perturbations upon a rapid mean flow in the longitudinal $x$ direction (with only a weak mean velocity gradient along the transverse $y$ direction). In that case, up to first order in $\frac{a}{\bar{u}}$ where $a$ is a typical amplitude of the velocity fluctuations and $\bar{u}$ is the mean surface velocity, equations (8) to (10) reduce to the following simple expression for the elevation:

$$
\frac{\eta=\bar{\eta}-\bar{u} u^{\prime}}{g}
$$


Superposed upon a mean surface level $\bar{\eta}$, the fluctuations in free-surface elevation scale linearly with the fluctuations in longitudinal velocity, by a factor equal to the mean velocity divided by the gravitational constant $g$. Qualitatively, conservation of energy head along streamlines results in particle velocities which are slowest at the crests and fastest at the troughs of the oscillatory flow, an observation which was already consigned be Lonardo Da Vinci in his notebooks some centuries ago [17]. In its quantitative version (11), the observation provides a way to extract from the horizontal velocity field an estimate of the local free-surface elevation relative to its average level. By pursuing the expansion to second order, an estimate of the error associated with the first order approximation can be made, and amounts to $\approx 1 \mathrm{~mm}$ for the conditions of the present experiments.

\section{B. Velocity field acquisition and averaging}

Before applying the above principle, velocity measurements are extracted from the high-frame rate image sequences by means of a pattern-based PTV algorithm. Particles are first detected on the images by applying a method similar to the one sketched above in section III.A. The Voronoï algorithm of Capart et al. is then used to track particles from one frame to the next by comparing the patterns associated with a local neighbourhood of the Voronoi diagram built upon particle centroids. This makes it possible to match particles over displacements which can be of the same order as the mean particle interdistance (whereas the simple nearest neighbour tracking methods of PTV require the displacement to be small with respect to this interdistance). In acquiring the surface velocity field, it is implicitly assumed that the floating tracers closely espouse the motion of the fluid at the free-surface. Due to the relatively large size of the tracers $(9 \mathrm{~mm})$, some deviations are however to be expected. These effects are difficult to estimate and are not accounted for in the method.

To regularise the raw velocity field and project it onto a regular grid, averages are performed first along particle trajectories (oriented in a predominantly longitudinal direction), then along the transverse direction. Top hat filters are used, choosing filter widths which are again calibrated to minimise the combined effects of attenuation and noise. Error levels of the order of $1 \mathrm{~mm}$ on the resulting elevation are estimated from both the trajectory and the transverse filtering. On the basis of the regularised velocity field, equation (10) is finally used to obtain the oscillatory elevation field component.

\section{COMPARISON OF RESULTS AND DISCUSSION}

Examples of final surfaces obtained by both methods are presented on Fig. 5. Since the velocimetric method cannot determine the mean water height, the latter has been substracted from the stereo results for ease of comparison. Because they result from a $2 \mathrm{D}$ binning which assigns an elevation even to areas where few particles were identified, the stereo results extend over the whole domain. The velocimetric results, on the other hand, are only given on the restricted domain covered by the retrieved trajectories of the particles.
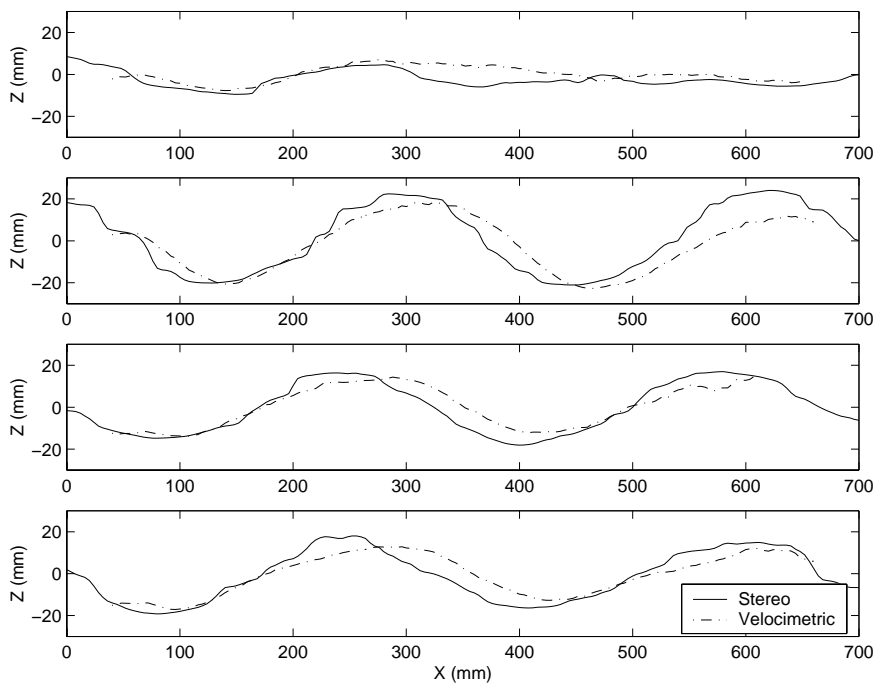

Figure 4: Comparison of surfaces cross-sections for run number 3. From top to bottom: $y=100, y=200, y=300$ and $y=400$.

The two methods were designed in such way as to be completely independent. It is thus reasonable to judge their global accuracy by comparing the results of both methods while keeping their respective accuracy in mind. Fig. 4 shows the strong correlation between the two methods: amplitude, wavelength and patterns of the antidunes are similar. However, some differences still exist between the two surfaces, mostly in the phase and in the local elevation of the surface.

The cause of the phase lag between the velocimetric and stereoscopic profiles could not be identified with certainty. As the velocimetric elevation lags behind the stereoscopic elevation, it is possible that this effect is associated with a delayed response of the floating tracers with respect to elevation changes. Wavebreaking at the crests could be another cause of discrepancy, while an inadevertent spatial bias of one of the steps of the algorithms cannot be completely excluded.

Some local discrepancies in amplitude can be ascribed to the surface reconstruction process used for stereovision. In zones of lower particle densities, Bayesian averaging is performed over a cell which can be larger than the relevant physical feature. A zone subject to this problem can be seen on surface 6 around position $(x, y)=(125,400)[\mathrm{mm}]$ (Fig. 5g). In this zone, the synchronisation interpolation and stereo matching appears to have broken down due to the local turbulence associated with wavebreaking. Consequently, the sharp crest was badly reconstructed as a crater.

The surfaces also exhibit textures which reflect the peculiarities of each of the two methods. Longitudinal stripes in the velocimetric results are the memories of the streamlines along which velocities are measured and averaged. The Bayesian averaging of the stereo method, on the other hand, results in staircase-like profile gradients. These are fine-grained effects, however, which do not overly affect the surface elevation maps.

Overall, the quality of the comparison is encouraging. Discrepancies in amplitude are of the order of the anticipated 


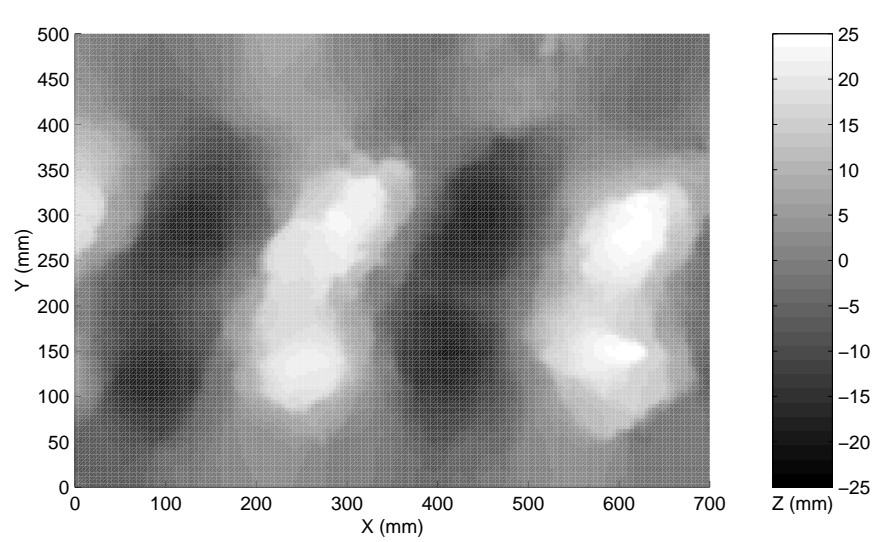

(a)

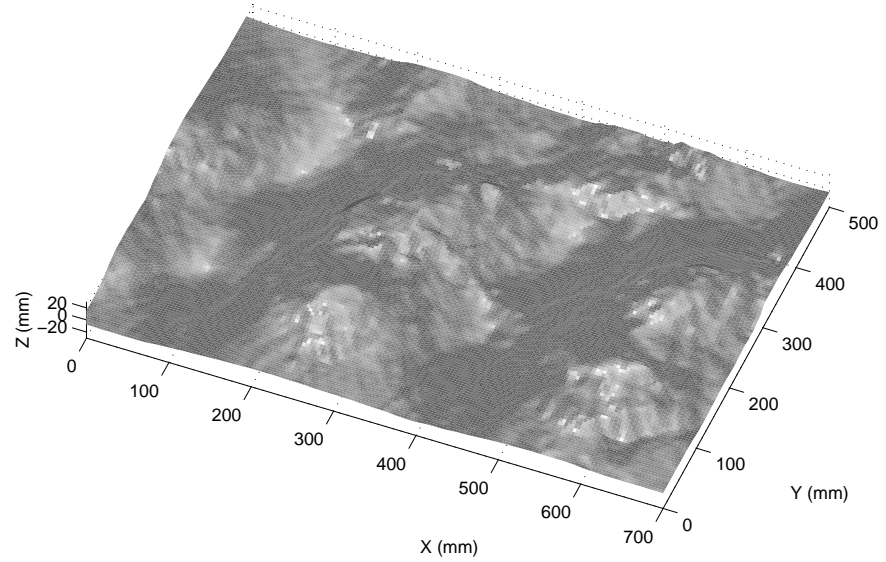

(c)

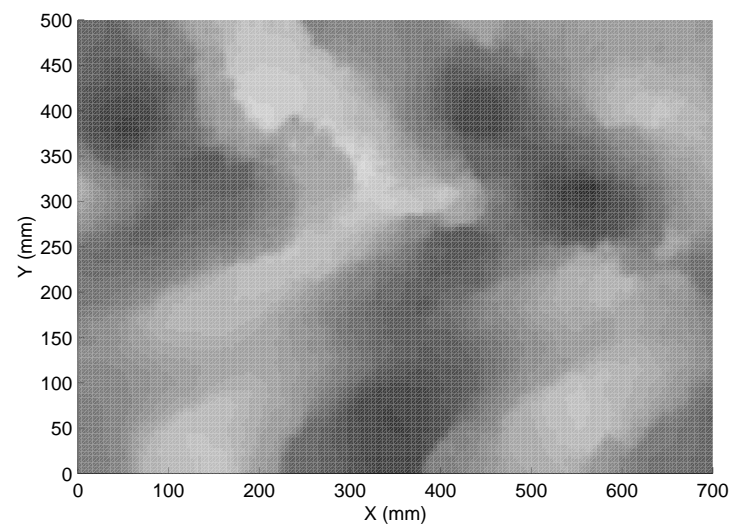

$(e)$

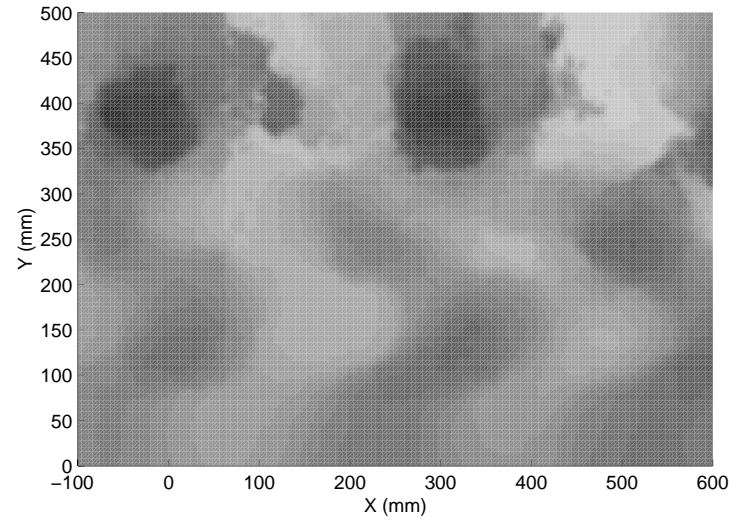

$(g)$

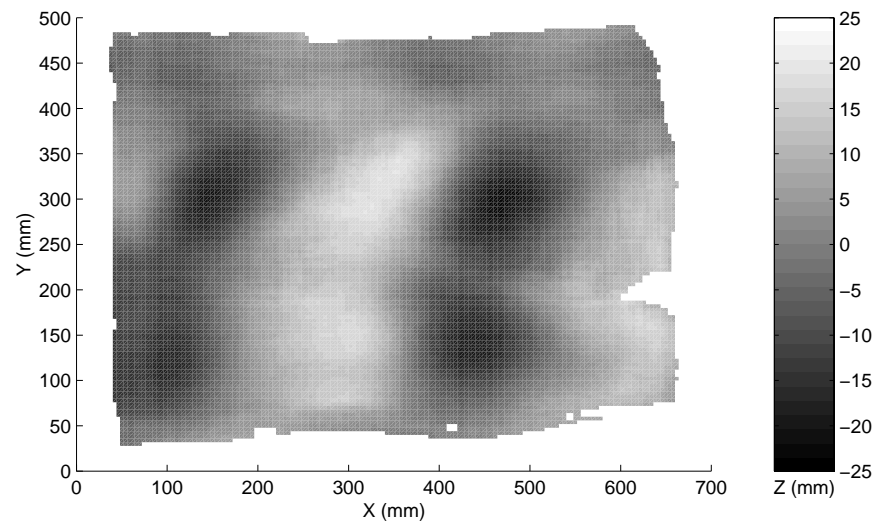

$(b)$

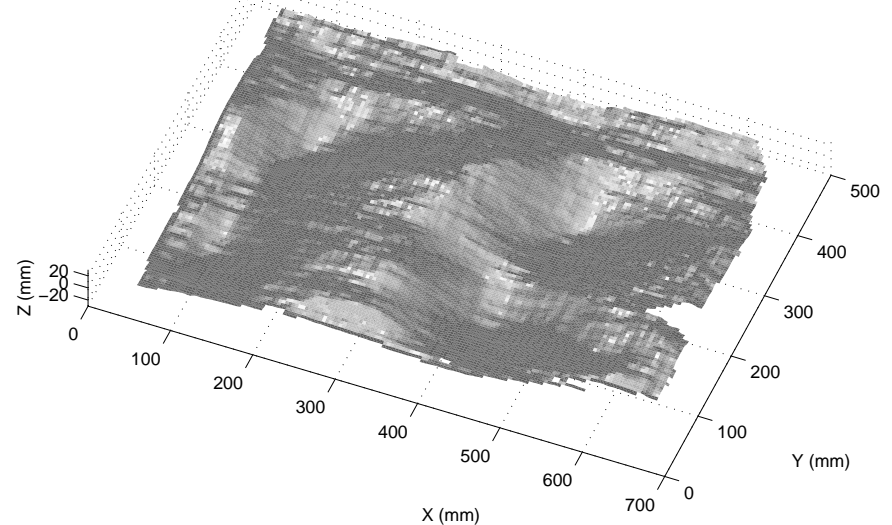

$(d)$

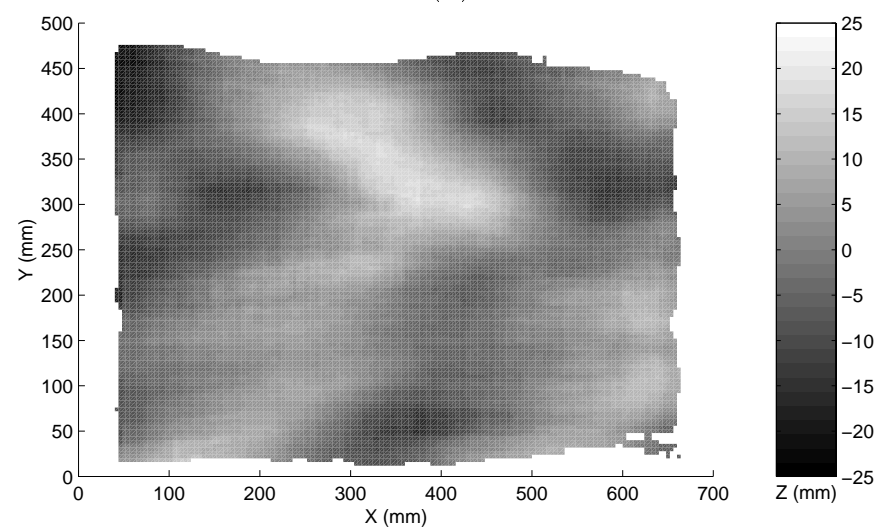

$(f)$

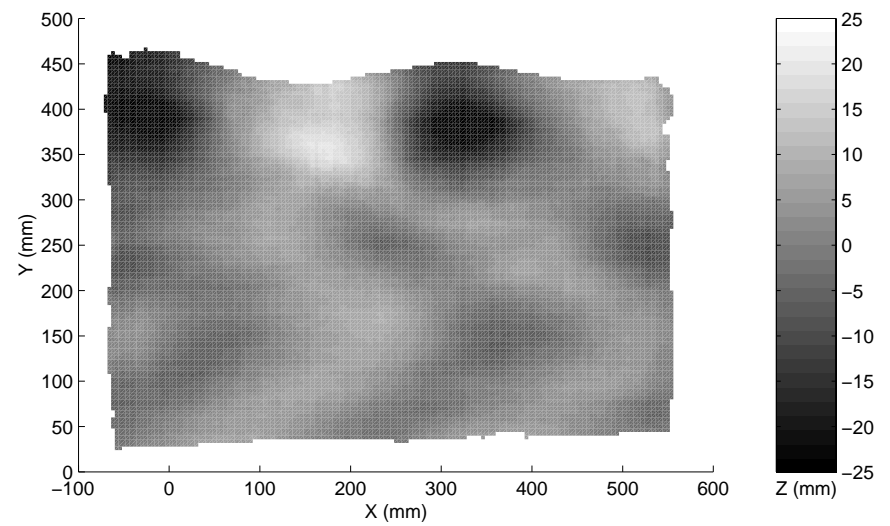

(h)

Figure 5: Results of the reconstruction for several surfaces. Left column present stereo reconstruction, right column velocimetric reconstruction. (a), (b): surface 3, depth map. (c), (d): surface 3 rendered. (e), (f): surface 5. (g), (h): surface 6. 
errors. The discrepancies highlighted in distorted $\mathrm{z}$-axis plots, moreover, appear much less severe when the surfaces are shown at their true aspect ratios (Fig. 5c). Most importantly, the spatial patterns are well-captured by both methods. The measurements vividly depict a variety of motifs, including narrow trains of peaks and throughs, broad crested rolls, and zig-zag patterns. All the surfaces also show some asymmetry in their patterns. Due to their continuous evolution and to the complex way in which light interacts with the rough water surface, such organised patterns cannot easily be observed by pure visual inspection of laboratory flows.

\section{CONCLUSIONS}

Two distinct imaging techniques were developed for the measurement of the oscillatory free-surface of flows over antidunes. Both measurement principles succeed in capturing antidune patterns, and the results compare favourably with each other. Beyond the particular application at hand, the results illustrate how stereoscopic and velocimetric matching algorithms can be exploited for the three-dimensional characterisation of flowing fluid surfaces. Future prospects include the use of synchronised high-resolution cameras and smaller particles in order to obtain more accurate, denser information. Also, application of the techniques to evolving antidune fields at a larger scale is contemplated.

\section{REFERENCES}

[1] J.F.Kennedy, "The mechanics of dunes and antidunes in erodible-bed channels," Journal of Fluid Mechanics, vol. 16, 1963 pp. $521-544$

[2] J.R.L.Allen, "Sedimentary structures-their character and physical basis," Amsterdam: Elsevier, 1984

[3] J.Alexander, C.Fielding, "Gravel antidunes in the tropical Burdekin River, Queensland, Australia," Sedimentology, vol. 44, 1997, pp. 327-337

[4] G.V.Middleton, "Antidune cross-bedding in a large flume," J. Sediment. Petrology, vol. 35, 1965, pp. 922-927

[5] R.J.Adrian, "Particle-imaging techniques for experimental fluid mechanics," Annu. Rev. Fluid Mechanics, vol. 23, 1991 pp. 261-304

[6] D.Douxchamps, "Stereometric Reconstruction of the Free Surface Associated to a Flow over Three-Dimensional Antidunes," Louvain-la-Neuve: Telecom. and Remote Sensing Lab., Bachelor Thesis, June 1998

[7] S. Mallat, "A Theory for Multiresolution Signal Decomposition: The Wavelet Representation," IEEE Trans. Pat. Anal. Mach. Intell., vol.11, July 1989 pp. 674-693

[8] I. Daubechies, "Orthonormal bases of compactly supported wavelets," Comm. Pure Appl. Math., vol.41, Nov. 1988 pp. 909-996

[9] R.N.Strickland, H.I.Hahn, "Wavelet Transform for Object Detection and Recovery," IEEE Trans. Image Proc., vol. 6, May 1997 pp. 724-735

[10] R.Jain, R.Kasturi, B.G.Schunck, "Machine Vision," New York: McGraw-Hill, 1995
[11] R.Y.Tsai, "A Versatile Camera Calibration Technique for High-Accuracy 3D Machine Vision Metrology Using Offthe-Shelf TV Cameras and Lenses," IEEE J. of Robotics and Auto., vol. 3, August 1987 pp.323-344

[12] H.Capart, D.L.Young, Y.Zech, "Voronoï imaging methods for the measurements of granular flows," submitted, 2000

[13] R.O.Duda, P.E.Hart, "Pattern classification and scene analysis," New York: Wiley-Interscience, 1973

[14] D.Devriendt, "Reconstruction tridimensionnelle de la surface d'un écoulement sur antidunes à partir de ses lignes de courant acquises par imagerie digitale," Louvain-laNeuve: Civil Eng. Dept., Bachelor Thesis, June 1998

[15] J.A.Liggett, "Fluid Mechanics," McGraw Hill College Div., 1994

[16] R.S.Johnson, "A Modern Introduction to the Mathematical Theory of Water Waves," Cambridge Texts in Applied Mathematics, 1997

[17] L.Da Vinci, "The Notebooks of Leonardo Da Vinci," Dover, 1975 